\title{
A Nanoporous Graphene/Nitrocellulose Membrane Beneficial to Wound Healing
}

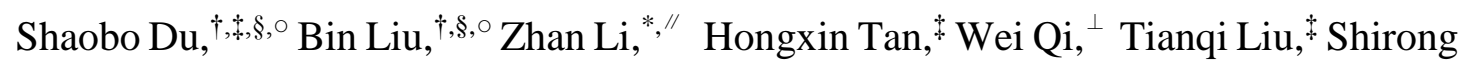
Qiang, ${ }^{\#}$ Taofeng Zhang, ${ }^{\dagger,}$ Fuxiang Song,${ }^{\dagger}{ }^{\S}$ Xiujuan Chen, ${ }^{\S}$ Jia Chen, ${ }^{\ddagger}$ Hongdeng Qiu, ${ }^{*},+, \nabla$ and Wangsuo $\mathrm{Wu}^{*}, \dagger$

${ }^{\dagger}$ School of Nuclear Science and Technology, Lanzhou University, Lanzhou 730000, China

tCAS Key Laboratory of Chemistry of Northwestern Plant Resources and Key Laboratory for Natural Medicine of Gansu Province, Lanzhou Institute of Chemical Physics, Chinese Academy of Sciences, Lanzhou 730000, China

${ }^{\S}$ School of Stomatology, Lanzhou University, Lanzhou 730000, China

"Frontier Science Center for Rare Isotopes, Lanzhou University, Lanzhou 730000, China

${ }^{\perp}$ Hubei Key Laboratory of Bioinorganic Chemistry \& Materia Medica, School of Chemistry and Chemical Engineering, Huazhong University of Science and Technology, Wuhan 430000, China

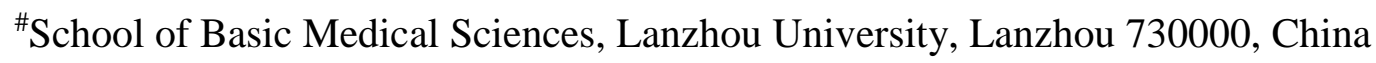
${ }^{\nabla}$ College of Chemistry, Zhengzhou University, Zhengzhou 450001, China

*Corresponding Authors: Zhan Li: liz@lzu.edu.cn; Hongdeng Qiu: hdqiu@licp.cas.cn; Wangsuo Wu:wuws@1zu.edu.cn 


\section{Characterizations of GO prepared by the Hummers' method}

The morphology and structure of GO were investigated through TEM, FT-IR spectra, XRD, and Raman spectra. The results showed that the prepared GO exhibits high performance (Figure S1).

(a)

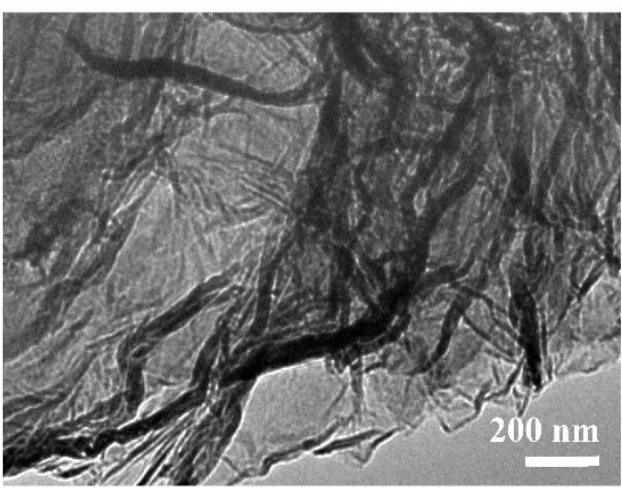

(c)

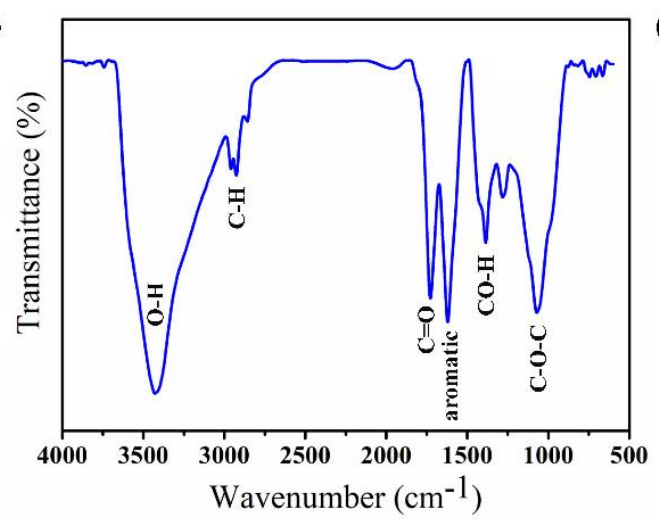

(b)
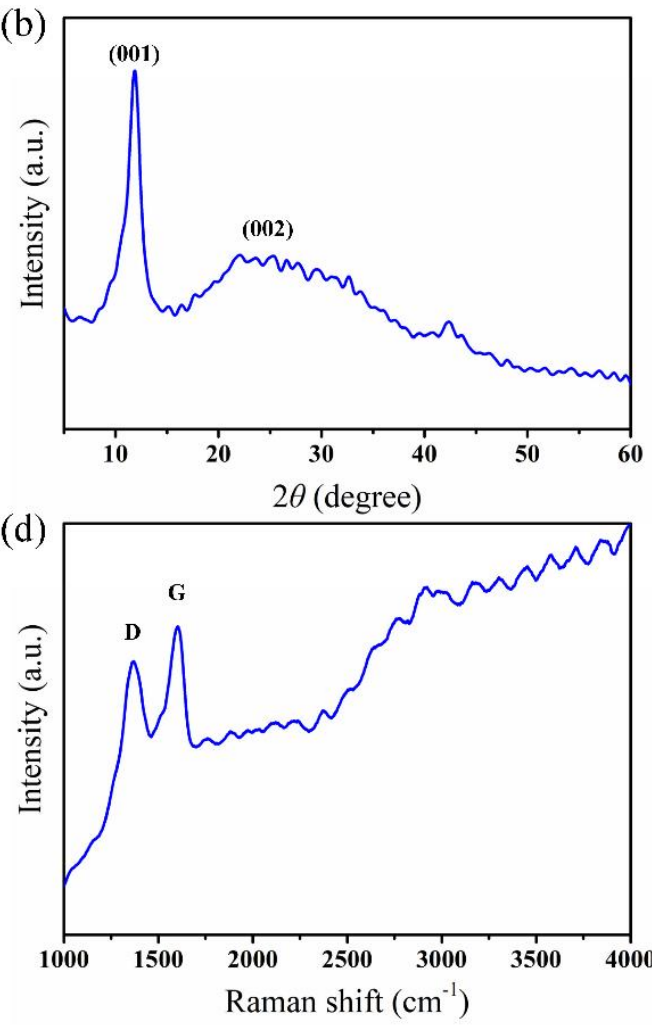

Figure S1. Characterization of graphene oxide (GO). (a) The morphology observation of GO by TEM. (b-d) XRD pattern, FT-IR spectra, and Raman spectra of GO.

\section{The O 1s spectrum of XPS and distribution of pore size for NPG}

The $\mathrm{O} 1 \mathrm{~s}$ spectrum indicates that the $\mathrm{C}-\mathrm{OH}$ peak located at $532.97 \mathrm{eV}$, and the $\mathrm{C}=\mathrm{O}$ peak located at $531.19 \mathrm{eV}$ (Figure $\mathrm{S} 2 \mathrm{a})^{1}$. The pore size of NPG was mainly distributed in $22.5-32.5 \mathrm{~nm}$ (Figure S2b). 

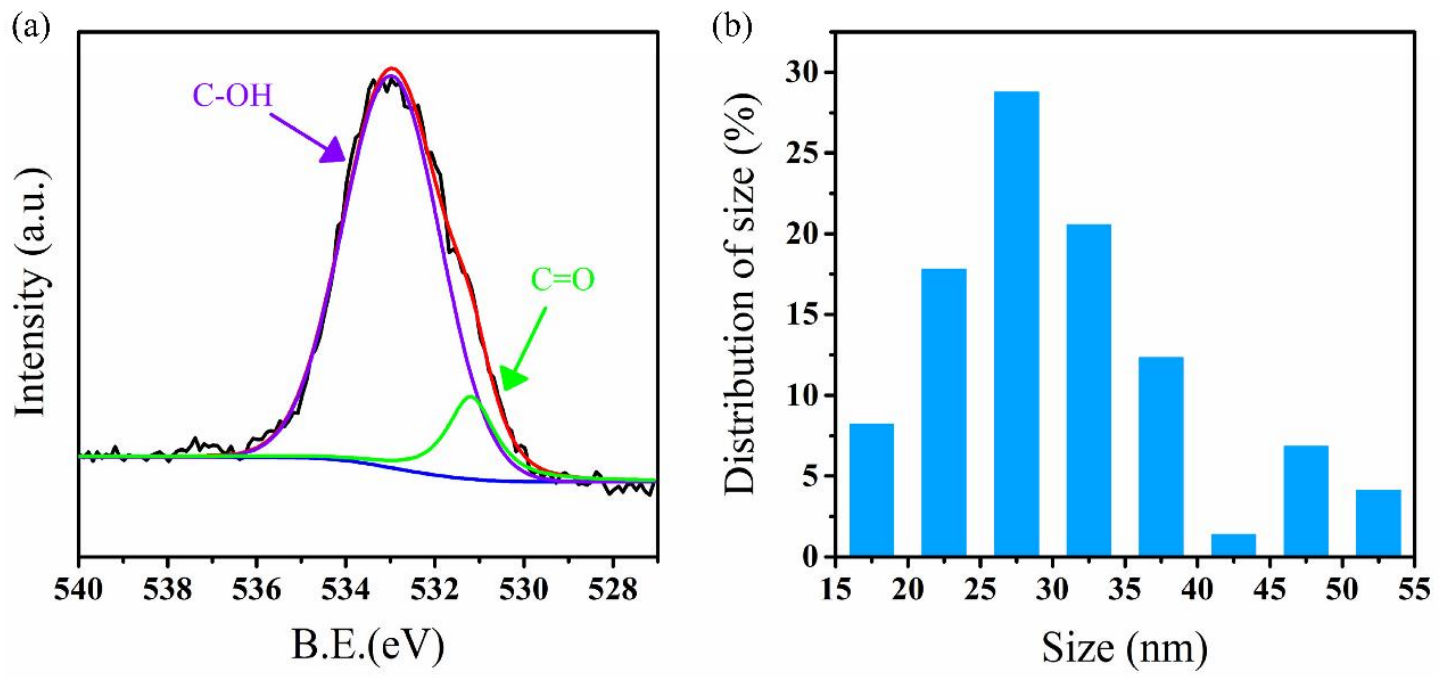

Figure S2. Characterization of nanoporous graphene (NPG). (a) O 1s spectra in XPS of NPG. (b)

Distribution of pore size for NPG. Distributions of pore size were calculated using software of Nano

Measurer 1.2.

\section{The morphology of the GON membranes and the NPGN membranes}

The photographs of the GON membranes were recorded using a digital camera, and the surface and cross-section morphology of the membranes were observed using SEM. The results showed that the GON membranes were brown compared to the black NPGN membrane. Moreover, the surface morphology of the GO membranes was similar to that of nitrocellulose membrane and the GO is less exposed on the membrane surface, and the cross section structure of the GON membrane is relatively compact (Figure S3a). The figure S3b showed that the distribution of NPG on the membrane surface gradually increased with the increase of its content. 
(a)
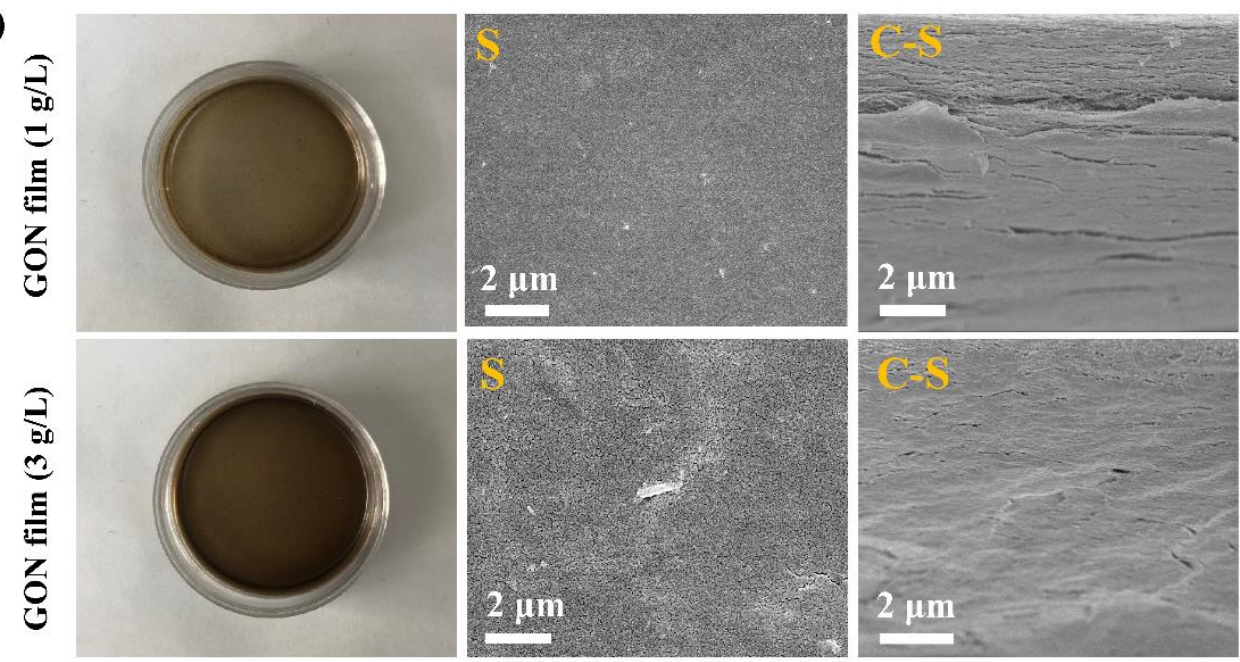

(b)
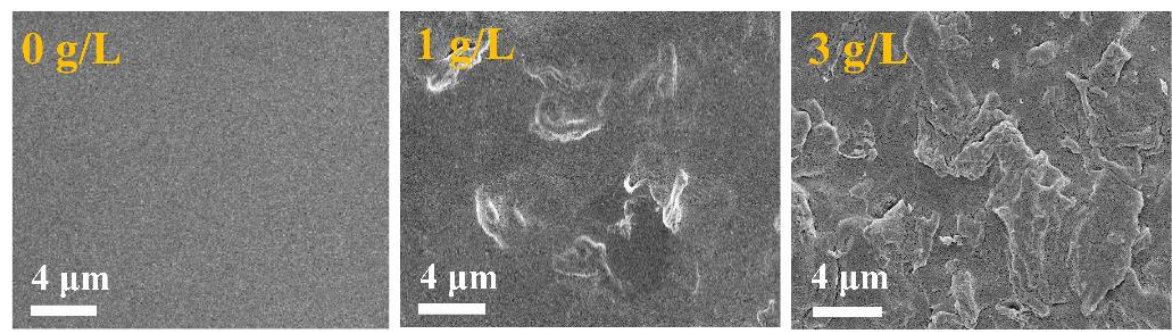

Figure S3. The morphology characterization of the GON membranes and the NPGN membranes.

(a) Membrane morphology of the GON membranes. Photographs of the GON membranes containing $1 \mathrm{~g} / \mathrm{L}$ and $3 \mathrm{~g} / \mathrm{L}$ of the GO respectively (the left column). The surface (S) morphology of the membranes were observed by scanning electron microscope (SEM; the middle column). The cross-section (C-S) morphology of the membranes were observed by SEM (the right column). (b) The surface (S) morphology of the NPGN membranes containing 0 , 1, and $3 \mathrm{~g} / \mathrm{L}$ of the NPG respectively were observed by SEM.

\section{The rising rates of WVTR for NPGN and GON membranes}


(a)

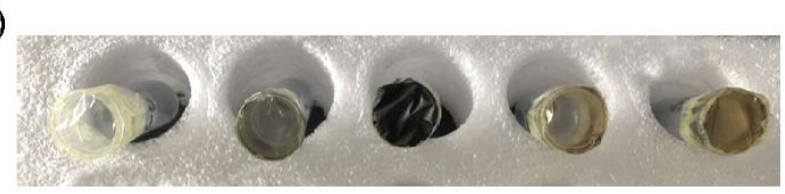

(b)

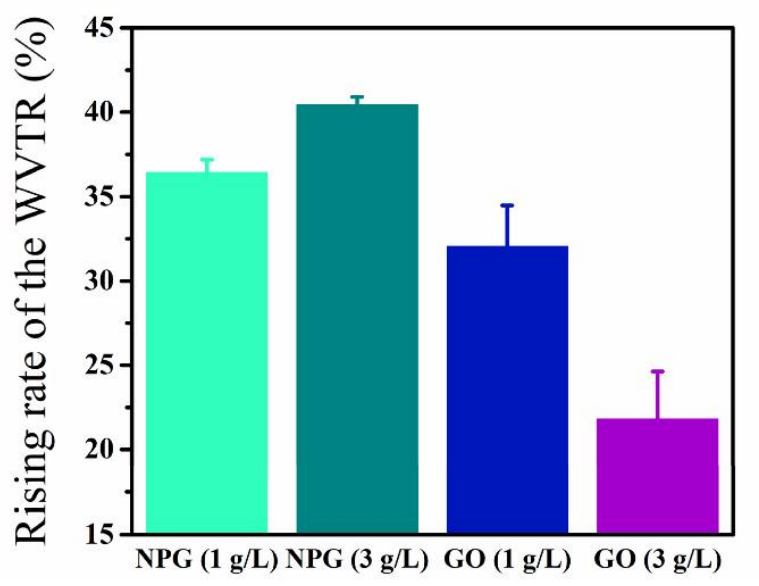

Figure S4. The rising rates of WVTR for NPGN membranes and GON membranes compared with nitrocellulose membrane. (a) Detection device photograph of WVTR for different membranes. From left to right: nitrocellulose membrane, NPGN membrane containing $1 \mathrm{~g} / \mathrm{L}$ NPG, NPGN membrane containing $3 \mathrm{~g} / \mathrm{L}$ NPG, GON membrane containing $1 \mathrm{~g} / \mathrm{L} \mathrm{GO}$, and GON membrane containing 3 g/L GO. (b) The rising rates of WVTR for NPGN membranes and GON membranes compared with nitrocellulose membrane: 1 g/L NPG: 36.43\%; 3 g/L NPG: 40.43\%; 1 g/L GO: 32.05\%; 3 g/L GO: $21.80 \%$

\section{Cellular biocompatibility of NPGN membrane for HeLa cells in vitro}

In addition to the human skin fibroblasts, the HeLa cells were also used for detecting the cellular biocompatibility of NPGN membrane by MTT assay. The HeLa cells were incubated with NPGN membranes for 1, 2, and 4 days, and the cell viabilities were analyzed. The results showed that both the nitrocellulose membrane and the NPGN membranes had a good biocompatibility. 


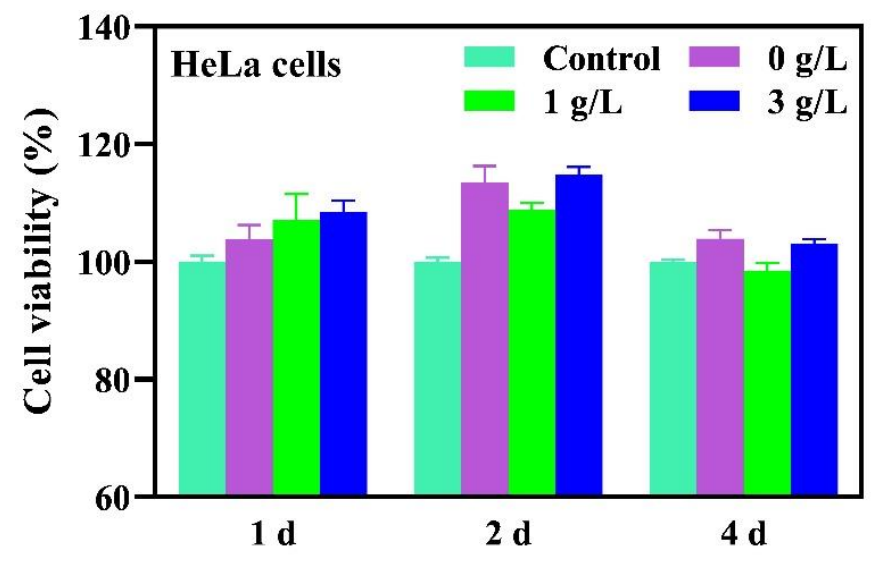

Figure S5. Cellular biocompatibility of the NPGN membranes for HeLa cells in vitro, the HeLa cells were respectively incubated for 1,2 , and 4 days with NPGN membranes.

\section{The band-aid of NPGN membrane in the mouse model}

(a)

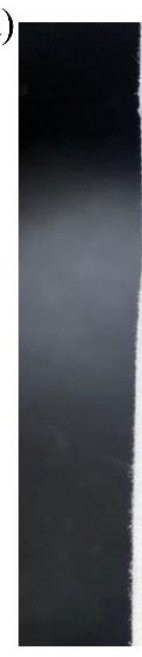

(b)

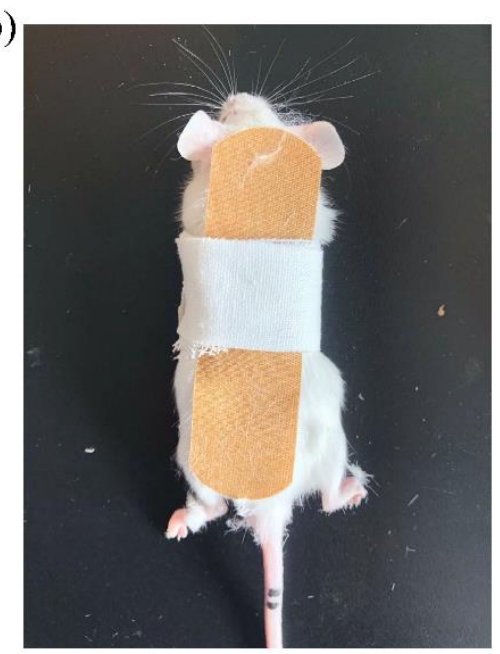

Figure S6. The band-aid made of NPGN membranes was used in a mouse full-thickness skin defect

wound model. (a) The band-aid made of NPGN membranes. (b) The mouse treated with the bandaid of NPGN membrane.

\section{The hematoxylin and eosin stained histological sections of the normal skin tissue}

It was found that more mature tissue structures were grown 7 days after surgery in 
the $3 \mathrm{~g} / \mathrm{L}$ group compared with control, $0 \mathrm{~g} / \mathrm{L}$ and $1 \mathrm{~g} / \mathrm{L}$ of groups. Even so, there still were some significant differences between it and normal skin tissue. The histological morphology of normal tissue was shown in Figure S7. The glands in $3 \mathrm{~g} / \mathrm{L}$ group were visibly less complex and mature than that in normal tissue, and the structures of fat layer and muscle layer disappeared in the wound healing groups.

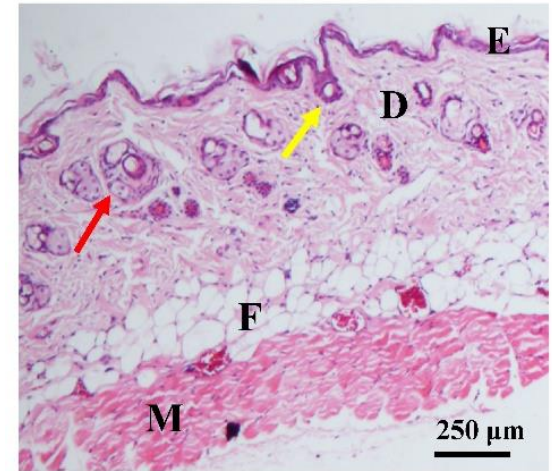

Figure S7. Representative image for hematoxylin and eosin-stained histological sections of the normal skin tissue. "E" indicates the epidermis, "D" indicates the dermis layer, "F" indicates the fat layer, and " $\mathrm{M}$ " indicates the muscle layer. The yellow arrow indicates the hair follicle and the red arrow indicates the gland.

\section{REFERENCES}

(1) Yang, D.; Velamakanni, A.; Bozoklu, G.; Park, S.; Stoller, M.; Piner, R. D.; Stankovich, S.; Jung, I.; Field, D. A.; Ventrice, C. A.; Ruoff, R. S. Chemical Analysis of Graphene Oxide Membranes after Heat and Chemical Treatments by X-Ray Photoelectron and Micro-Raman Spectroscopy. Carbon 2009, 47, 145-152. 